\title{
CP-Logic Theory Inference with Contextual Variable Elimination and Comparison to BDD Based Inference Methods
}

\author{
Wannes Meert, Jan Struyf and Hendrik Blockeel \\ Dept. of Computer Science, Katholieke Universiteit Leuven, Belgium \\ $\{$ Wannes.Meert, Jan.Struyf, Hendrik.Blockeel\}@cs.kuleuven. be
}

\begin{abstract}
There is a growing interest in languages that combine probabilistic models with logic to represent complex domains involving uncertainty. Causal probabilistic logic (CP-logic), which has been designed to model causal processes, is such a probabilistic logic language. This paper investigates inference algorithms for CP-logic; these are crucial for developing learning algorithms. It proposes a new CP-logic inference method based on contextual variable elimination and compares this method to variable elimination and to methods based on binary decision diagrams.
\end{abstract}

\section{Introduction}

Formalisms from the field of Statistical Relational Learning (SRL) combine concepts from two fields, uncertainty theory and (first-order) logic, to model complex worlds that cannot be represented by using techniques from one of these fields alone. SRL uses uncertainty theory to express the uncertainty that is present in everyday life, while it employs logic to represent complex relations between the objects in the world.

Fast inference algorithms are key to the success of SRL. Inference speed is not only important while answering probabilistic queries, but it is also crucial for developing fast parameter and structure learning algorithms [1]. Uncertainty theory and logic each have their own specific set of inference algorithms. SRL models can be queried by performing both types of inference sequentially [2-4]. However, as the field matured, more efficient solutions have been presented in which the two are more interleaved, that is, logic inference is used while performing probabilistic inference and not only as a preprocessing step [5-7].

In this paper, we focus on the SRL language CP-logic [8], which is a language that has been designed to model causal processes. Since CPlogic was introduced, several inference algorithms have been proposed for (a subset of) CP-logic. This paper proposes a new CP-logic inference method, which more closely integrates logic an probabilistic inference. 
The method is based on Poole and Zhang's Contextual Variable Elimination (CVE) [9]. CVE is a Bayesian network (BN) inference algorithm that extends traditional Variable Elimination (VE) [10] by factorizing each factor in the factor representation of the BN further into a product of so-called confactors. This confactor representation compactly represents both the logical and probabilistic component of a (ground) CP-logic theory. By exploiting this compact representation, CVE can be orders of magnitude faster than VE on a given inference task.

This paper is organized as follows. In Section 2, we explain the basic notions behind CP-logic. Section 3 presents an overview of the known CPlogic inference techniques. We introduce CP-logic inference by means of CVE in Section 4. Section 5 presents an experimental comparison among the different inference methods. We end with a conclusion and a number of directions for future work in Section 6 .

\section{CP-Logic}

In many applications, the goal is to model the probability distribution of a set of random variables that are related by a causal process, that is, the variables interact through a sequence of non-deterministic or probabilistic events. Causal probabilistic logic (CP-logic) [8] is a probabilistic logic modeling language that can model such processes. The model takes the form of a CP-logic theory (CP-theory), which is a set of CP-events in which each event is represented as a rule of the following form:

$$
\left(h_{1}: \alpha_{1}\right) \vee \ldots \vee\left(h_{n}: \alpha_{n}\right) \leftarrow b_{1}, \ldots, b_{m} .
$$

with $h_{i}$ atoms, $b_{i}$ literals, and $\alpha_{i}$ causal probabilities; $0<\alpha_{i} \leq 1, \sum \alpha_{i} \leq$ 1 . We call the set of all $\left(h_{i}: \alpha_{i}\right)$ the head of the event, and the conjunction of literals $b_{i}$ the body. If the body of a CP-event evaluates to true, then the event will happen and cause at most one of the head atoms to become true; the probability that the event causes $h_{i}$ is given by $\alpha_{i}$ (if $\sum \alpha_{i}<1$, it is also possible that nothing is caused).

It is part of the semantics [8] of CP-logic that each rule independently of all other rules makes one of its head atoms true when triggered. CPlogic is therefore particularly suited for describing models that contain a number of independent stochastic events or causal processes. 
Example. The CP-theory

$$
\begin{array}{rlrl}
\text { shops }(\text { john }): 0.2 & \leftarrow . & & \left(c_{1}\right) \\
\text { shops }(\text { mary }): 0.9 & \leftarrow . & \left(c_{2}\right) \\
(\text { spaghetti }: 0.5) \vee(\text { steak }: 0.5) & \leftarrow \text { shops }(\text { john }) . & & \left(c_{3}\right) \\
\text { (spaghetti }: 0.3) \vee(\text { fish }: 0.7) & \leftarrow \text { shops }(\text { mary }) . & & \left(c_{4}\right)
\end{array}
$$

models the situation that John and his partner Mary may independently decide to go out to buy food for dinner. The causal probability associated with each meal indicates the probability that the fact that John (respectively Mary) goes shopping causes that particular meal to be bought.

It may be tempting to interpret the CP-theory parameters as the conditional probability of the head atom given the body, e.g., based on

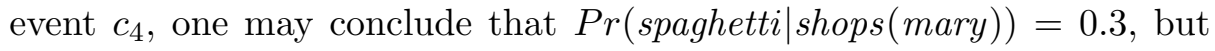
this is incorrect. The correct value is $0.3+0.2 \cdot 0.5 \cdot 0.7=0.37$ since Mary buys spaghetti with probability 0.3 , but there is also a probability of $0.2 \cdot 0.5 \cdot 0.7$ that the spaghetti is bought by John $(0.2 \cdot 0.5)$ and not by Mary (0.7). Thus, for head atoms that occur in multiple events, the mathematical relationship between the CP-theory parameters and conditional probabilities is somewhat complex, but it is not unintuitive. The meaning of the causal probabilities in the events is quite simple: they reflect the probability that the body causes the head to become true. This is different from the conditional probability, but among the two, the former is the more natural one to express. Indeed, the former is local knowledge: an expert can estimate the probability that shops(mary) causes spaghetti without considering any other possible causes for spaghetti.

CP-logic is closely related to other probabilistic logics such as ProbLog [11], Independent Choice Logic (ICL) [12], Programming in Statistical Modelling (PRISM) [13], and Bayesian Logic Programs (BLPs) [2]. Meert et al. [1] compares CP-logic to these other formalisms.

\section{Known CP-Theory Inference Methods}

Probabilistic inference in the context of SRL is the process of computing the conditional probability $\operatorname{Pr}(q \mid e)$, with $q$ the query atom and $e$ a conjunction of literals, which is known as the evidence. The example from the previous section, which illustrated how to compute $\operatorname{Pr}$ (spaghetti |shops(mary)), is as such an example of probabilistic inference.

A simple general probabilistic inference method for CP-logic is to directly apply the process semantics of CP-logic as defined by Vennekens et al. [8]. This is, however, not a computationally efficient method and 
faster alternatives have been proposed in the mean time. These alternative methods are discussed in the following sections.

\subsection{Variable Elimination}

Blockeel and Meert $[14,1]$ propose a transformation that can transform any acyclic CP-theory with a finite Herbrand universe into a particular BN, which they call the Equivalent Bayesian Network (EBN) of the CPtheory. Based on this transformation, CP-theory inference is performed by first transforming the CP-theory into its EBN and by subsequently running a traditional BN inference algorithm, such as Variable Elimination (VE) [10] on the EBN to answer the probabilistic query.

In this section, we recall the transformation procedure. (For the proof of correctness we refer to Blockeel and Meert [14]). We only consider ground CP-theories, so a non-ground CP-theory must be grounded first. The following three steps construct the EBN of a ground CP-theory (Fig. 1 shows the resulting EBN for the "shopping" example):

1. Create for every atom in the CP-theory a so-called atom node in the EBN, and for every event a so-called choice node. Next, create for each event in the CP-theory the following edges. Create for every head atom of the event an edge from the event's choice node towards the atom's atom node, and for every literal in the event's body an edge from the literal's atom node towards the event's choice node.

2. A choice node can take $n+1$ values, where $n$ is the number of head atoms in the CP-event that it represents. It takes the value $i$ if the $i^{\text {th }}$ head atom is chosen by the probabilistic process. It takes the value 0 if none of the head atoms is chosen (this can happen if $\sum \alpha_{i}<1$ ). The node's CPT is filled out as follows. Given that the body of the event is true, the probability that the node takes the value $i \neq 0$ is precisely the causal probability $\alpha_{i}$ given in the event's head. The probability that it takes the value 0 is equal to $1-\sum \alpha_{i}$. If the body is not true, then the choice node takes the value 0 with probability 1.0.

3. An atom node is a Boolean node of which the CPT represents the deterministic OR function of the different ways in which the atom can be caused by a CP-event.

\subsection{ProbLog}

This section explains how to perform CP-theory inference by transforming the CP-theory into ProbLog [5], which is a probabilistic programming 


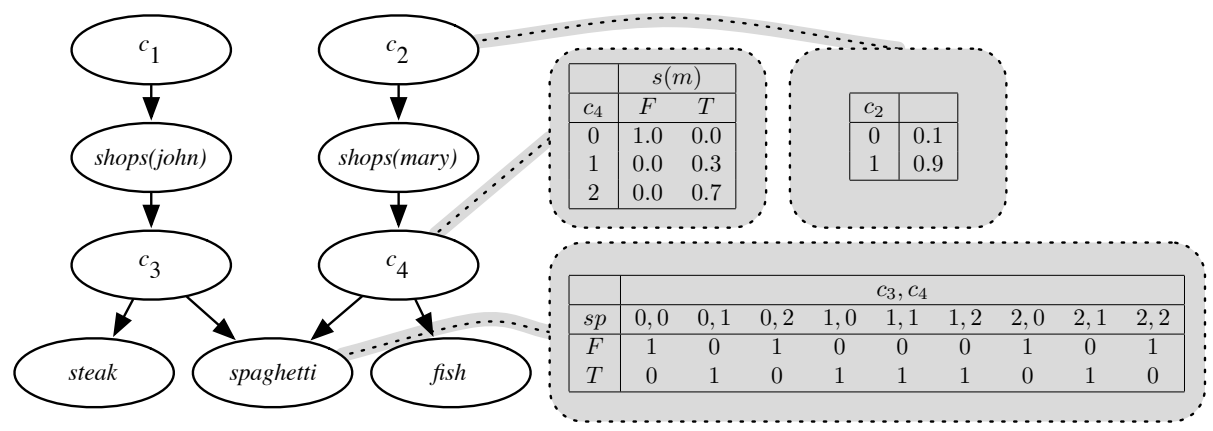

Fig. 1. EBN for the "shopping" example. The CPT for event $c_{4}$ represents the probability distribution over its possible outcomes $\left(c_{4}=0 \ldots 2\right)$ conditioned on its body atom shops(mary). The CPT for the atom node spaghetti represents the deterministic OR $c_{3}=1 \vee c_{4}=1$ (spaghetti is selected by the third event or by the fourth).

language with an efficient inference engine, and by subsequently relying on ProbLog's inference.

A ProbLog program consists of a set of probabilistic facts and a set of Prolog clauses (Fig. 2, first step). That is, it can be seen as a CP-theory in which all events have precisely one head atom and zero or more positive body literals, and in which the probability of an event's head atom is equal to 1.0 if its body is non-empty.

ProbLog can serve as a target language to which other probabilistic logic modeling languages can be compiled. In particular, acyclic CPtheories without negation can be translated into ProbLog. Fig. 2 illustrates how the CP-theory from the "shopping" example can be translated into ProbLog. The translation introduces probabilistic facts to encode which of the head atoms are chosen (their names start with "c_"). In the example, one probabilistic fact for each event suffices. Besides the probabilistic facts, the ProbLog program includes for each CP-event $e$ one clause for each head atom $h$ of the event. This clause has $h$ as head and its body is a conjunction based on the probabilistic fact for $e$ and the body of $e$ (in this case, the body includes either the probabilistic fact or its negation). For events that have more than two possible outcomes, more than one probabilistic fact is required.

ProbLog's inference engine works as follows. Given a query, it first computes all proofs of the query (using standard Prolog SLD resolution) and collects the probabilistic facts used in these proofs in a DNF formula. The second step of Fig. 2 shows the resulting DNF for the query 


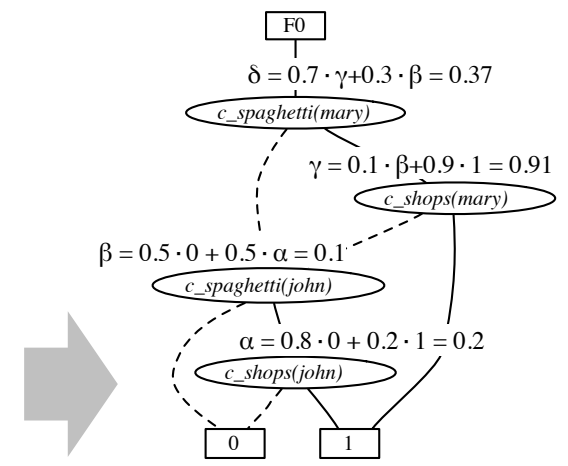

shops(john): $1 \leftarrow c_{-}$shops $($john $)$.

shops(mary): $1 \leftarrow c_{-}$shops(mary).

spaghetti: $1 \leftarrow$ c_spaghetti(john), shops(john).

steak: $1 \leftarrow$ not (c_spaghetti(john)), shops(john).

spaghetti: $1 \leftarrow c_{-}$spaghetti(mary), shops(mary).

fish: $1 \leftarrow$ not(c_spaghetti(mary)), shops(mary).

$($ c_shops(john) ^ c_spaghetti(john $))$

$\vee\left(c \_s h o p s(\right.$ mary $) \wedge c \_s p a g h e t t i($ mary $\left.)\right)$

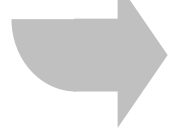

Prolog clauses

Fig. 2. Steps performed to calculate the probability of the query $\operatorname{Pr}$ (spaghetti) for a ProbLog program equivalent to the shopping CP-theory.

$\operatorname{Pr}($ spaghetti); each disjunct corresponds to one proof of "spaghetti". Next, it converts this DNF formula to a binary decision diagram (BDD).

A BDD (Fig. 2, right) is a compact representation for a Boolean formula (in this case, the DNF). It is a rooted directed acyclic graph, which consists of decision nodes and two terminal nodes called 0-terminal and 1-terminal. Each decision node is labeled with an atom from the formula and has two outgoing edges: left and right. The left subgraph represents the case that the atom is false and the right subgraph represents the case that it is true. A path from the root to the 1-terminal represents a value assignment that makes the formula true, or in the case of ProbLog it represents a proof of the program.

Relying on this BDD representation, ProbLog computes the query's probability in one bottom-up pass through the BDD (using dynamic programming). To do so, it exploits the fact that the left and right subgraphs of a node represent mutually exclusive cases: the left subgraph represents the cases that the atom is false and the right subgraph the cases that it is true. As a result, the probability of the query can be computed by recursively using the formula $\operatorname{Pr}\left(n_{i}\right)=\left(1-c_{i}\right) \operatorname{Pr}\left(\operatorname{left}\left(n_{i}\right)\right)+c_{i} \operatorname{Pr}\left(\operatorname{right}\left(n_{i}\right)\right)$, with $\operatorname{Pr}(n)$ the probability computed for node $n, c_{i}$ the probability listed in the program for probabilistic fact $i$, and $\operatorname{left}(n)(\operatorname{right}(n))$ the left (right) child of $n$. The resulting $\operatorname{Pr}(n)$ values are indicated at the top of each node in Fig. 2. Further details can be found in [5].

\section{3 cplint}

Inspired by ProbLog, Riguzzi [15] proposes cplint, which is a CP-theory inference system that makes use of BDDs in a similar way as ProbLog. 
There are two differences with the transformation to ProbLog. First, cplint uses a different encoding to represent which head atom is caused by a CP-event. Second, cplint supports negation. When it encounters a negative body literal $\neg a$ in a proof, it computes all proofs of $a$ and includes the negation of the DNF resulting from all these proofs into the original DNF. This process is recursive and can be computationally expensive.

\section{Inference with Contextual Variable Elimination}

As said before, a CP-theory can be transformed to an EBN. However, a CP-theory may contain more structural information than a $\mathrm{BN}$, and this logical structure has to be encoded numerically in the EBN's factors. This may result in factors with redundant information (a factor may have many identical columns) and cause suboptimal inference. This effect can be seen in the factor for spaghetti in Fig. 1. To address this problem, we propose to use Contextual Variable Elimination (CVE) [9].

\subsection{Contextual Variable Elimination}

CVE makes use of a more specific form of conditional independence known as contextual independence [16,9].

Definition 1 (Contextual Independence). Assume that $\mathbf{x}, \mathbf{y}, \mathbf{z}$ and $\mathbf{c}$ are sets of variables. $\mathbf{x}$ and $\mathbf{y}$ are contextually independent given $\mathbf{z}$ and context $\mathbf{c}=\mathbf{C}$, with $\mathbf{C} \in \operatorname{dom}(\mathbf{c})$, iff

$$
\operatorname{Pr}\left(\mathbf{x} \mid \mathbf{y}=\mathbf{Y}_{\mathbf{1}} \wedge \mathbf{z}=\mathbf{Z}_{\mathbf{1}} \wedge \mathbf{c}=\mathbf{C}\right)=\operatorname{Pr}\left(\mathbf{x} \mid \mathbf{y}=\mathbf{Y}_{\mathbf{2}} \wedge \mathbf{z}=\mathbf{Z}_{\mathbf{1}} \wedge \mathbf{c}=\mathbf{C}\right)
$$

for all $\mathbf{Y}_{\mathbf{1}}, \mathbf{Y}_{\mathbf{2}} \in \operatorname{dom}(\mathbf{y})$ for all $\mathbf{Z}_{\mathbf{1}} \in \operatorname{dom}(\mathbf{z})$ such that $\operatorname{Pr}\left(\mathbf{y}=\mathbf{Y}_{\mathbf{1}} \wedge \mathbf{z}=\right.$ $\left.\mathbf{Z}_{\mathbf{1}} \wedge \mathbf{c}=\mathbf{C}\right)>0$ and $\operatorname{Pr}\left(\mathbf{y}=\mathbf{Y}_{\mathbf{2}} \wedge \mathbf{z}=\mathbf{Z}_{\mathbf{1}} \wedge \mathbf{c}=\mathbf{C}\right)>0$. We also say that $\mathbf{x}$ is contextually independent of $\mathbf{y}$ given $\mathbf{z}$ and context $\mathbf{c}=\mathbf{C}$.

VE represents the joint distribution as a product of factors. CVE factorizes the joint distribution further by replacing each factor by a product of contextual factors or confactors. A confactor $r_{i}$ consists of two parts: a context and a table:

$$
<\underbrace{v_{1} \in V_{1 i} \wedge \ldots \wedge v_{k} \in V_{k i} \wedge \ldots \wedge v_{n} \in V_{n i}}_{\text {context }}, \quad \underbrace{\operatorname{factor}_{i}\left(v_{k}, \ldots, v_{m}\right)}_{\text {table }}>
$$

The context is a conjunction of set membership tests $\left(v_{j} \in V_{j i}, V_{j i} \subseteq\right.$ domain $\left(v_{j}\right)$ ), which indicates the condition under which the table is applicable. It is used to factorize factors into confactors based on Def. 1. 
The table stores probabilities for given value assignments for a set of variables $\left(v_{k}, \ldots, v_{m}\right)$. In the original CVE algorithm, the context was limited to equality tests. Our implementation also allows set membership tests, which are required to concisely represent CP-theories (e.g., to represent the inequality tests in the contexts in Fig. 3, left).

The set of confactors that together represent the conditional probability distribution (CPD) of a variable $v$ is mutually exclusive and exhaustive. This means that for each possible value assignment $v=V, \mathbf{p a}(v)=$ $\mathbf{V}_{\mathbf{p}}$, with $\mathbf{p a}(v)$ the parents of $v$, there is precisely one confactor of which the table includes the parameter $\operatorname{Pr}\left(v=V \mid \mathbf{p a}(v)=\mathbf{V}_{\mathbf{p}}\right)$. These conditions ensure that the product of all confactors is identical to the product of the original factors and thereby equal to the joint distribution.

We describe the CVE algorithm at a high level (the complete algorithm can be found in [9]). Similar to VE, CVE eliminates the non-query non-evidence variables one by one from the joint distribution. To eliminate a variable, it relies on three basic operations: (a) multiplying two confactors with identical contexts; (b) summing out a variable that appears in the table of a confactor; and (c) summing out a variable that appears in the contexts. The operations have as pre-condition that no other compatible confactor exists with a different context. Two contexts are incompatible if there exists a variable that is assigned different values in the contexts; otherwise they are compatible. All three operations are only possible if (parts of) the contexts are identical. To satisfy this precondition, CVE uses an operation called splitting. Given two compatible confactors, repeated splitting can be used to create two confactors with identical contexts, on which operations (a)-(c) can be performed. These basic operations are repeatedly executed until all non-query non-evidence variables are eliminated; the resulting distribution is the answer to the query.

Confactors may represent CPDs more compactly than tables, but as the previous discussion illustrates, this comes at the cost of more complicated basic operations.

\subsection{Converting a CP-Theory to a Set of Confactors}

As seen in Section 3, a CP-theory can be represented as an EBN, in which the CPDs are represented by tables. In the previous section, we saw that confactors may lead to a more compact representation. Here, we define the transformation that constructs such a representation. The structure of the EBN is the same as before, but the CPDs are now sets of confactors. 


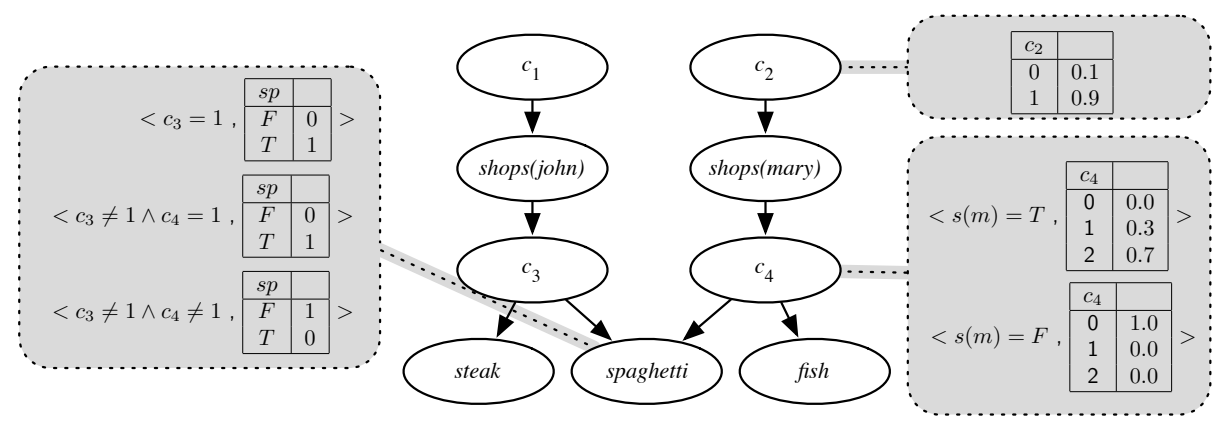

Fig. 3. Confactor representation for node spaghetti (left) and $c_{4}$ (right)

The transformation consists of the following two steps (Fig. 3 shows the result for the "shopping" example):

1. The CPD for a choice node is represented by multiple confactors. The context of one confactor represents the case that the event's body is true. The other confactors constitute the case that the body is false, and make the set of confactors mutually exclusive and exhaustive.

For example, the first confactor for $c_{4}$ (Fig. 3, right) represents the case that the body shops (mary) is true and the event chooses to make one of the head atoms true $\left(c_{4}=1\right.$ for spaghetti, $c_{4}=2$ for fish). The other $c_{4}$ confactor corresponds to the case that shops(mary) is false; in that case no head atom is caused $\left(c_{4}=0\right)$.

2. The CPD of an atom node is factorized into multiple confactors that together encode an OR-function (by means of the contexts). If at least one of the events where the atom is in the head has selected the atom, it becomes true; otherwise, it will be false.

The above transformation can be extended to improve inference efficiency. For example, we represent a set of CP-events with the same atoms in the head and mutually exclusive bodies by a single choice node. Since such a set of CP-events represents a decision tree this can be encoded by confactors in a compact manner (see Fig. 4). The choice node indicates the outcome of the decision tree and every leaf of the decision tree is converted into a confactor with as context the conjunction of conditions on the path from the root to the leaf.

Also, a CPD is not further factorized if the resulting confactors are not more compact (in terms of the number of parameters) than the original table representation (e.g., in our implementation, $c_{4}$ 's CPD is not split into two confactors as in Fig. 3, but kept as a single factor). 


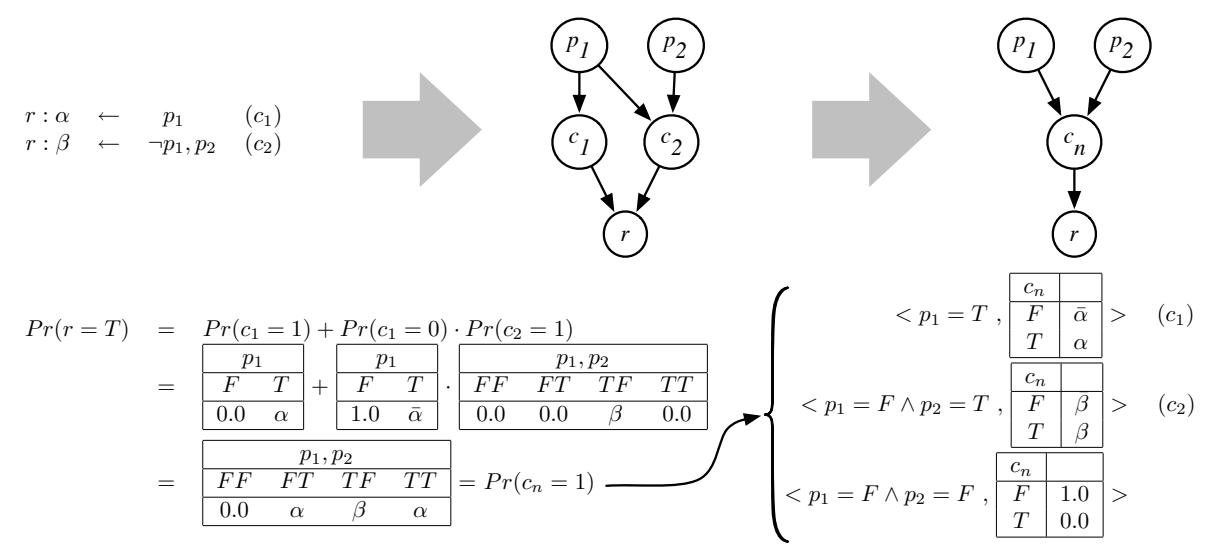

Fig. 4. Example showing how to combine multiple CP-events $\left(c_{1}\right.$ and $\left.c_{2}\right)$ with mutually exclusive bodies into a single choice node $\left(c_{n}\right)$. The probability $\operatorname{Pr}(r=T)$ can be rewritten such that it depends on $c_{n}$. The CPD for $c_{n}$ combines the distributions for $c_{1}$ and $c_{2}$ in such a way that the confactors that represent this CPD have the same structure in their contexts as the original set of CP-events has in its bodies $(\bar{\alpha}=1-\alpha)$.

Once the set of confactors that represents the CP-theory is constructed, we use the CVE algorithm [9] to perform CP-theory inference.

\section{$5 \quad$ Experiments}

\section{$5.1 \quad$ Setup}

We evaluate the inference methods on the task of inferring the marginal distribution of one designated variable in four CP-theories of varying complexity. We always select the variable with the highest inference cost and do not include any evidence (i.e., we consider the most difficult case). The theories are available at http://www.cs.kuleuven.be/ dtai/cplve/ilp09. Fig. 5 presents the results. Graphs (b) and (d) do not include results for ProbLog because they include negation. In the following paragraphs we discuss the used theories.

Growing head. For every atom $a_{i}, i>0$ there is a probabilistic fact that causes the atom to become true. Besides these probabilistic facts, there is also a CP-event for every atom $a_{i}$ in which $a_{i}$ is in the body and all $a_{j}$, $j<i$ are in the head. In other words, atom $a_{i}$ can cause all other atoms $a_{j}$ for which $j<i$. This results in an EBN that has a large number of Noisy-OR structures and these are heavily interconnected. 
The CP-theory of size 4 is given below as an example (the size parameter indicates the number of atoms in the theory):

$$
\begin{aligned}
a 0: 1.0 & \leftarrow a 1 . & & a 1: 0.5 \leftarrow . \\
a 0: 0.5 \vee a 1: 0.5 & \leftarrow a 2 . & & a 2: 0.5 \leftarrow . \\
a 0: 0.33 \vee a 1: 0.33 \vee a 2: 0.33 & \leftarrow a 3 . & & a 3: 0.5 \leftarrow .
\end{aligned}
$$

Growing body with negation. This CP-theory models a set of decision trees in which the outcome of one tree is used as input by other trees. Each decision tree is represented by a set of CP-events. The theory of size 4 is given below as an example.

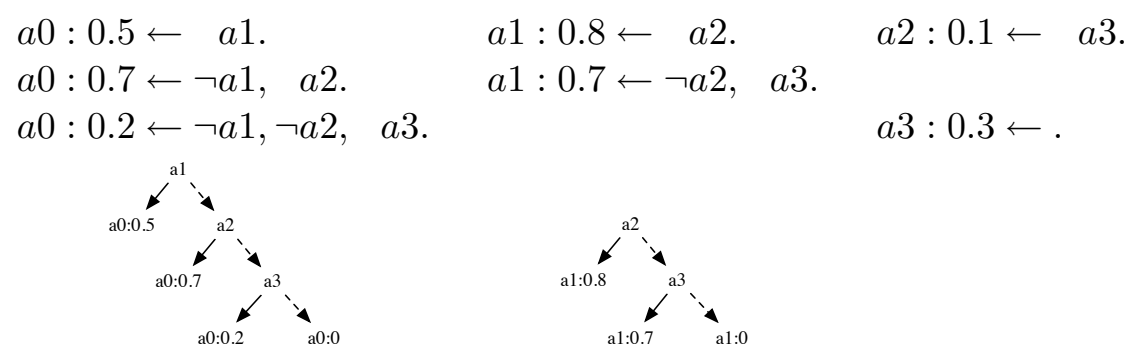

The events that share the same head together form a decision tree because their bodies are mutually exclusive. Inference methods, such as CVE, that exploit that the bodies of such events represent a decision tree can use this knowledge to improve inference efficiency (Section 4.2).

Blood group [2] is a Bayesian network with multi-valued variables and models the probability that a person $(p)$ has a specific blood type $(b t)$ given the chromosomes ( $p c$ and $m c$ ) that he inherited from his parents (which are again probabilistically dependent on the blood types of their parents). We convert this $\mathrm{BN}$ to a CP-theory and perform inference on the resulting theory. We represent each multi-valued variable as a predicate with two arguments: the first argument is an identifier that identifies the person and the second argument is instantiated to one of the possible values of the multi-valued variable. The variable's CPT is encoded by several CP-events. The head of such an event encodes the distribution over the variable's values given a particular value assignment, encoded in the event's body, for the variable's parents in the BN.

A fragment of the theory of size 4 is given below. 


$$
\begin{aligned}
p c(p, a): 0.3 \vee p c(p, b): 0.3 \vee p c(p, n i l): 0.4 & \leftarrow \\
m c(p, a): 0.3 \vee m c(p, b): 0.3 \vee m c(p, n i l): 0.4 & \leftarrow \\
& \ldots \\
b t(p, a): 0.9 \vee b t(p, b): 0.03 \vee b t(p, a b): 0.03 & \leftarrow p c(p, a), m c(p, a) . \\
b t(p, a): 0.03 \vee b t(p, b): 0.03 \vee b t(p, a b): 0.9 & \leftarrow p c(p, b), m c(p, a) .
\end{aligned}
$$

$U W$-CSE. This data set [17] records information about the University of Washington's department of Computer Science and Engineering. For this experiment, we start from a model that was learned from this data by means of ordering search [18] and convert this model into a CP-theory. The structure of the resulting theory is complex and contains a combination of the structures of previous examples; it includes both Noisy-OR and decision trees. Note that this theory and also Blood Type are first order theories. We use a simple grounder based on Prolog's SLD resolution to create the relevant grounding for the given probabilistic query.

\section{$5.2 \quad$ Results}

For theory (a), the BDD based inference methods (cplint and ProbLog) are faster than CVE and VE for large problem instances. CVE and VE are slower partly because they compute the probability that a variable is true, but also the probability that it is false (separately). It is well known that for Noisy-AND, which occurs in the ProbLog program that theory (a) is transformed into (see Fig. 2), it is more efficient to only compute the probability $P_{T}$ that its outcome is true and to calculate the probability that it is false as $1-P_{T}$. This explains the advantage of the BDD based methods on this theory: they only compute the probability that an atom is true.

The results for theory (b) do not include measurements for ProbLog because ProbLog's inference engine does not support this type of CPtheories. ProbLog only allows negation on predicates that are defined in terms of probabilistic facts; it does not support negation on predicates that are defined by non-trivial clauses. However, the latter type of negation is required to express the decision trees that are modeled by this CP-theory. Supporting full negation in ProbLog is ongoing work (A. Kimmig, personal communication).

Theory (b) contains sets of CP-events that together express decision trees. Since CVE can combine such CP-events compactly into a set of confactors (see Fig. 4) it is more efficient than the other methods. More- 
(a) Growing Head

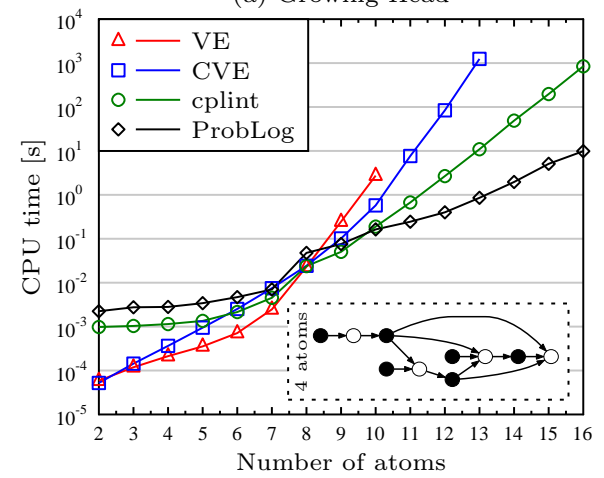

(c) Blood Group - bloodtype(p,a)

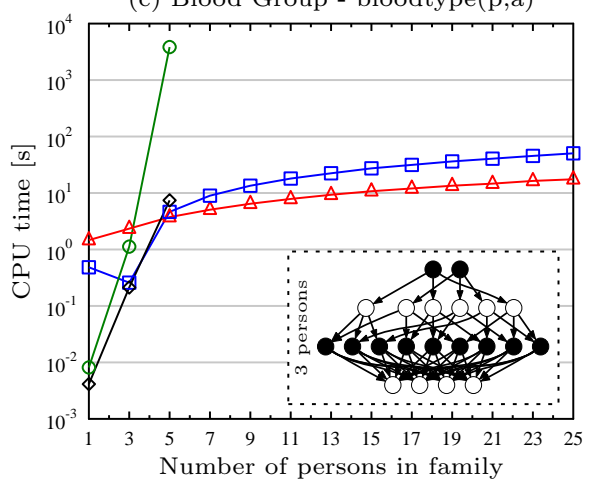

(b) Growing Body with Negation

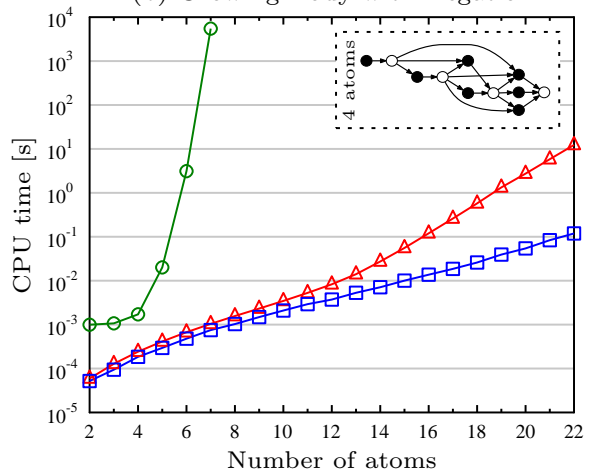

(d) UW-CSE - taught_by $(\mathrm{c}, \mathrm{p})$

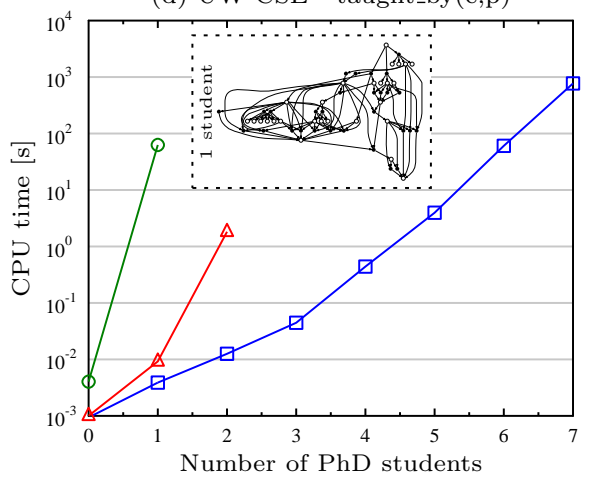

Fig. 5. Experimental results (including example EBNs for small theories)

over, CVE and VE outperform the BDD based method cplint due to the complexity of cplint's method for handling negation.

Theory (c) models a Bayesian network in CP-logic. Because CVE has more complex basic operations and tries to represent additional contextual independencies it is slower than VE for large theories in this case. VE and CVE both outperform the BDD based methods. The BDD methods' lower performance is partly due to their inability to represent a BN that has no extra structure beyond conditional independence in its most compact form, which is with tables. Another reason is that the same atom will be encountered multiple times in ProbLog's proofs; if that happens then the DNF formula will contain an identical subexpression for each occurrence. Computing all these subexpressions will require repeatedly proving the same goal and may lead to a larger BDD and to slower inference. Some of these redundant computations can be avoided by 'tabling' proofs [19, 
20]. Preliminary experiments, however, have shown that tabling does not always improve inference time.

Theory (d) includes a combination of the structures found in theories (a)-(c): it contains Noisy-OR relations, decision trees, and atoms appear repeatedly in proofs. CVE outperforms all other methods in this most general case.

CVE outperforms VE for large problem instances on theories (a), (b), and $(d)$. This is due to the compact representation with confactors instead of factors. VE runs out of memory after size 10 in (a) and size 2 in (d).

\section{Conclusion and Future Work}

We have proposed a new CP-theory inference method that transforms the given CP-theory to a set of confactors and then performs inference by running contextual variable elimination (CVE) on this representation. CVE outperforms VE with regard to both time and memory consumption for most large problem instances. Depending on the theory, CVE may also be faster than current BDD based methods.

In future work, we plan to incorporate (some of) the above inference methods into CP-theory learning algorithms. Second, we would like to investigate lifted inference for CP-theories. Known lifted-inference methods employ VE; we will try to extend this to CVE. A third item of interest is to investigate inference and learning methods for cyclic CP-theories.

Acknowledgments: Institute for the Promotion of Innovation through Science and Technology in Flanders (IWT-Vlaanderen) to WM. Research Fund K.U.Leuven to JS. GOA/08/008 'Probabilistic Logic Learning'. The authors are grateful to A. Kimmig and D. Fierens for the fruitful discussions and to F. Riguzzi for his suggestions about cplint.

\section{References}

1. Meert, W., Struyf, J., Blockeel, H.: Learning ground CP-logic theories by leveraging Bayesian network learning techniques. Fundamenta Informaticae 89(1) (2008) $131-160$

2. Kersting, K., De Raedt, L.: Bayesian logic programming: Theory and tool. In: Statistical Relational Learning. MIT Press (2007) 291-322

3. Haddawy, P.: Generating Bayesian networks from probability logic knowledge bases. In: 10th Conference on Uncertainty in Artificial Intelligence (UAI). (1994) 262-269

4. Wellman, M.P., Breese, J., Goldman, R.: From knowledge bases to decision models. Knowledge Engineering Review 7 (1992) 35-53 
5. Kimmig, A., Santos Costa, V., Rocha, R., Demoen, B., De Raedt, L.: On the efficient execution of ProbLog programs. In: Proceedings of the International Conference on Logic Programming (ICLP). (2008) 175-189

6. de Salvo Braz, R., Amir, E., Roth, D.: Lifted first-order probabilistic inference. In: Statistical Relational Learning. MIT Press (2007) 433-452

7. Singla, P., Domingos, P.: Lifted first-order belief propagation. In: Proceedings of the 23th AAAI Conference on Artificial Intelligence. (2008) 1094-1099

8. Vennekens, J., Denecker, M., Bruynooghe, M.: Representing causal information about a probabilistic process. In: Proceedings of the European Conference on Logics in Artificial Intelligence (JELIA). (2006) 452-464

9. Poole, D., Zhang, N.: Exploiting contextual independence in probabilistic inference. Journal of Artificial Intelligence Research 18 (2003) 263-313

10. Zhang, N., Poole, D.: A simple approach to bayesian network computations. In: 10th Biennial Canadian Artificial Intelligence Conference. (1994) 171-178

11. De Raedt, L., Kimmig, A., Toivonen, H.: ProbLog: A probabilistic Prolog and its application in link discovery. In: Proceedings of the 20th International Joint Conference on Artificial Intelligence (IJCAI). (2007)

12. Poole, D.: The independent choice logic for modelling multiple agents under uncertainty. Journal of Artificial Intelligence 94(1-2) (1997) 7-56

13. Sato, T., Kameya, Y.: 6. In: New advances in logic-based probabilistic modeling by PRISM. Probabilistic Inductive Logic Programming (2007)

14. Blockeel, H., Meert, W.: Towards learning non-recursive LPADs by transforming them into Bayesian networks. In: Proceedings of the 17th International Conference on Inductive Logic Programming (ILP). (2007) 94-108

15. Riguzzi, F.: A top down interpreter for LPAD and CP-logic. In: Proceedings of the 10th Congress of the Italian Association for Artificial Intelligence (AI*IA). (2007)

16. Boutilier, C., Friedman, N., Goldszmidt, M., Koller, D.: Context-specific independence in Bayesian networks. In: 12th Conference on Uncertainty in Artificial Intelligence (UAI96). (1996) 115-123

17. Richardson, M., Domingos, P.: Markov logic networks. Machine Learning 62(1-2) (2006) 107-136

18. Ramon, J., Croonenborghs, T., Fierens, D., Blockeel, H., Bruynooghe, M.: Generalized ordering-search for learning directed probabilistic logical models. Machine Learning 70(2-3) (March 2008) 169-188

19. Riguzzi, F.: The SLGAD procedure for inference on Logic Programs with Annotated Disjunctions. In: Proceedings of the 15th RCRA workshop on Experimental Evaluation of Algorithms for Solving Problems with Combinatorial Explosion. (2009)

20. Mantadelis, T., Janssens, G.: Tabling relevant parts of SLD proofs for ground goals in a probabilistic setting. In: International Colloquium on Implementation of Constraint and LOgic Programming Systems (CICLOPS). (2009) 